\title{
Fuzzy-Based Indoor Positioning by Using the Neighbor Points
}

\author{
Chih-Yung Chen, Shen-Whan Chen, Yu-Ju Chen, and Rey-Chue Hwang
}

\begin{abstract}
This paper presents a fuzzy-based indoor positioning system (IPS) by using the information of neighbor points to estimate the location of object. An 8x8 square meters indoor area was used as the experimental area. In the experimental field, the received signal strength (RSS) of 288 points, 392 points, 440 points and 704 points were sensed and collected by a hexagonal positioning station which is composed of six printed-circuit board SPARKLAN AX-106M antennas and Zigbee module. The sensed RSS values are then used to be the information of fuzzy system for the object's position estimation. From the experimental results shown, the proposed IPS and fuzzy estimation method do have the accurate positioning performance and indeed has the potential in the real application.
\end{abstract}

Index Terms-Fuzzy, IPS, neighbor points, RSS.

\section{INTRODUCTION}

Due to the rapid development of wireless communication techniques and personal networks, IPS has been widely used to locate people and objects in the indoor environments and several survey reports are also presented [1]-[3]. For instance, in large shopping malls, airports and hospitals, IPS can be used for navigation. IPS can also be used as the rescue tool while the disaster was happened in a large building. It is able to help the rescue team to find those people who need the help.

A complete IPS is mainly composed of two parts, i.e., the positioning estimation algorithm and the sensing hardware infrastructure. The positioning estimation algorithm is a calculation method for determining the object's location. So far, triangulation [4], [5], scene analysis [6], [7] and proximity [8], [9] are three main estimation algorithms used as the positioning works. Each algorithm has its specific advantages and disadvantages. For sensing hardware infrastructure, many wireless communication technologies have been wildly used in the sensing system of IPS. However, due to the demand of different functions and equipment, the sensing hardware infrastructure can be classified as wireless local area network (WLAN) [10]-[12], wireless sensor

Manuscript received June 15, 2018; revised August 5, 2018. This research was partially supported by the Ministry of Science and Technology, Taiwan, R.O.C. under Contracts MOST-106-2221-E-214-046.

Chih-Yung Chen is with the Computer and Communication Department, Shu-Te University, Kaohsiung, Taiwan (e-mail: mikechen@stu.edu.tw ).

Shen-Whan Chen is with the Department of Communication Engineering, I-Shou University, Kaohsiung, Taiwan (e-mail: jasonchen@isu.edu.tw).

Yu-Ju Chen is with the Department of Information Management, Cheng-Shiu University, Kaohsiung, Taiwan (e-mail: yjchen@csu.edu.tw).

Rey-Chue Hwang is with the Department of Electrical Engineering, I-Shou University, Kaohsiung, Taiwan (Corresponding author; tel.: 886-7-6577711; e-mail: rchwang@isu.edu.tw). network (WSN) [13], [14], radio frequency identification (RFID) [15], [16], Bluetooth [17], [18] and Zigbee [19], [20], etc.

In recent years, due to the application of expert experience becomes increasingly important, fuzzy theory has been widely used in different fields. It is a theory which can formulate human knowledge in a systematic manner and put it into the mechanism developed for a specific using. Thus, fuzzy theory used for IPS development has also been studied in several articles [21]-[27].

In this research, a fuzzy-based IPS is used to perform the object's location estimation. The positioning station composed of six printed-circuit board SPARKLAN AX-106M antennas and Zigbee modules is used to sense the RSS signals [28]. The detailed IPS hardware, fuzzy calculation model and positioning experiments will be described in the following sections.

\section{IPS HARDWARE INFRASTRUCTURE}

In our study, IPS is developed based on the wireless sensing network technology. The whole IPS includes positioning antenna array design, signal strength acquisition, reference database construction and positioning algorithm [29]. The positioning sector antenna array is composed of six SPARKLAN AX-106M antennas. The Zigbee module is used for transmitting and receiving signals. The sector antenna array is shown in Fig. 1. The whole IPS structure is presented in Fig. 2 which includes IPS station and positioning device.

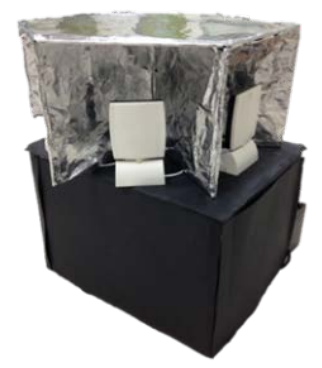

Fig. 1. The sector antenna array.

\section{FUZZy Estimation Algorithm}

It is known that fuzzy theory was initialized by Professor Lotfi Zadeh in 1965. It has been widely applied into many fields including the various engineering and social science applications [30]. Basically, a fuzzy system is a knowledge-based or rule-based system. Its heart is a knowledge base consisting of many fuzzy IF-THEN rules. 
The IF-THEN rule can be treated as an IF-THEN statement which standards an expert knowledge or experience.

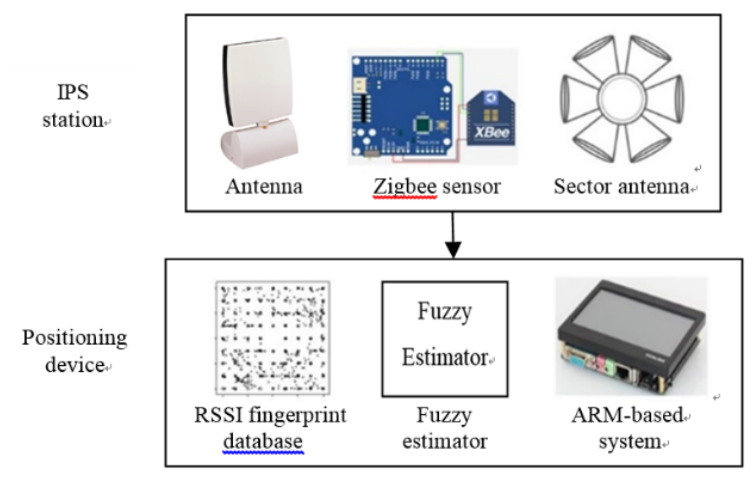

Fig. 2. IPS structure.

A completed fuzzy system is mainly composed of four parts, i.e. fuzzifier, fuzzy rule base, fuzzy inference engine and defuzzifier. The fuzzifer plays the role to make the crisp information become the fuzzy information. It generates a mapping from a real-valued point to a fuzzy set. Contrary, defuzzifer plays the role to make the fuzzy information become the crisp information. It is a mapping from a fuzzy set to a real-valued point. The fuzzy rule base is composed of a set of fuzzy IF-THEN rules. Each rule is created based on the knowledge or experience of the expert. It is the heart of the whole fuzzy system. The fuzzy inference engine can be treated as a fuzzy logic principle. It combines the fuzzy IF-THEN rules in rule base to obtain an appropriate mapping from a fuzzy set in fuzzifier to a fuzzy set of defuzzifier. Fig. 3 shows the fuzzy estimator developed for our IPS.

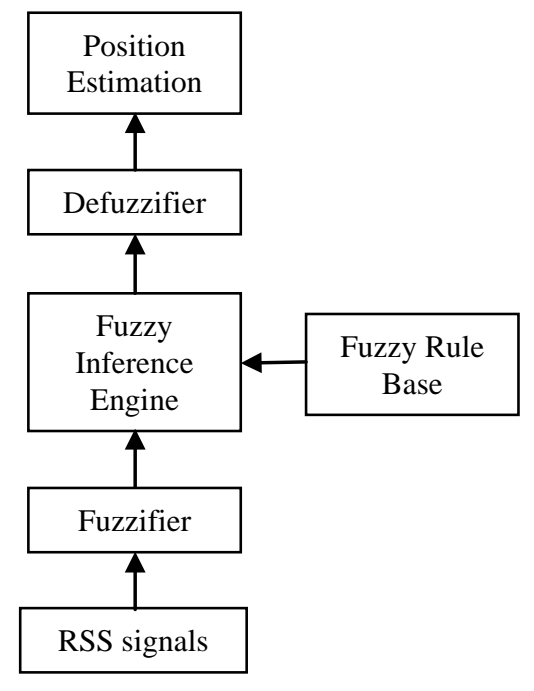

Fig. 3. Fuzzy estimator.

In our study, the coordinate of object is expected to be estimated by fuzzy IPS. At here, let $\mathbf{C}$ be a set of class vectors, i.e. IPS reference database, which is given by

$$
\mathbf{C}=\left\{\begin{array}{l}
\left(\left(x_{1}, y_{1}\right),\left(R_{11}, R_{12}, \ldots, R_{16}\right)\right), \\
\left(\left(x_{2}, y_{2}\right),\left(R_{21}, R_{22}, \ldots, R_{26}\right)\right), \\
\ldots,\left(\left(x_{m}, y_{m}\right),\left(R_{m 1}, R_{m 2}, \ldots, R_{m 6}\right)\right)
\end{array}\right\}
$$

$\left\{\left(x_{1}, y_{1}\right),\left(x_{2}, y_{2}\right), \ldots,\left(x_{m}, y_{m}\right)\right\}$ are the coordinates of reference positions and $\left\{\left(R_{11}, R_{12}, \ldots, R_{16}\right), \ldots,\left(R_{m 1}, R_{m 2}, \ldots\right.\right.$, $\left.R_{m 6}\right)$ \} are six RSS signals sensed on the reference nodes. Now, we assume RSS signal vector sensed at a blind node $\left(x_{b}, y_{b}\right)$ is $\left(\hat{R}_{b 1}, \hat{R}_{b 2}, \ldots, \hat{R}_{b 6}\right)$, then the fuzzy rule base can be constructed by the following $m$ fuzzy IF-THEN rules.

$$
\begin{aligned}
& \quad R u^{(1)}: \text { IF } \hat{R}_{b 1} \text { is } R_{11} \text { and } \hat{R}_{b 2} \text { is } R_{12} \text { and } \ldots \text { and } \hat{R}_{b 6} \text { is } R_{16} \text {, } \\
& \operatorname{THEN}\left(x_{b}=x_{1}, y_{b}=y_{1}\right) \text {. } \\
& \quad R u^{(2)}: I F \hat{R}_{b 1} \text { is } R_{21} \text { and } \hat{R}_{b 2} \text { is } R_{22} \text { and } \ldots \text { and } \hat{R}_{b 6} \text { is } R_{26} \text {, } \\
& \operatorname{THEN}\left(x_{b}=x_{2}, y_{b}=y_{2}\right) \text {. } \\
& \text {. } \\
& \quad R u^{(m)}: \text { IF } \hat{R}_{b 1} \text { is } R_{m 1} \text { and } \hat{R}_{b 2} \text { is } R_{m 2} \text { and } \ldots \text { and } \hat{R}_{b 6} \text { is } R_{m 6} \text {, } \\
& \operatorname{THEN}\left(x_{b}=x_{m}, y_{b}=y_{m}\right) \text {. }
\end{aligned}
$$

If there are $\mathrm{N}$ points in the reference database, then $m=1$, $2, \ldots, N$. In the fuzzy mechanism, the fuzzifier is Gaussian function which can be expressed by

$$
\mu\left(R_{m i}, \sigma\right)=\exp \left(-\frac{\left(\hat{R}_{b i}-R_{m i}\right)^{T}\left(\hat{R}_{b i}-R_{m i}\right)}{2 \sigma^{2}}\right), i=1,2, \ldots, 6
$$

$\sigma$ is the smoothing parameter of Gaussian function.

The fuzzy inference engine is the combination of the product inference engine with algebraic product for $t$-norm operator. The fuzzy relation inference value then can be calculated by

$$
\mu\left(R_{m}, \sigma\right)=\mu\left(R_{m 1}, \sigma\right) * \mu\left(R_{m 2}, \sigma\right) * \ldots * \mu\left(R_{m 6}, \sigma\right)
$$

The center average defuzzifier listed as follows is used to estimate the coordinate values of object position.

$$
\begin{gathered}
x_{b}=\frac{\sum_{m=1}^{N} x_{m} \mu\left(R_{m}, \sigma\right)}{\sum_{m=1}^{N} \mu\left(R_{m}, \sigma\right)} \\
y_{b}=\frac{\sum_{m=1}^{N} y_{m} \mu\left(R_{m}, \sigma\right)}{\sum_{m=1}^{N} \mu\left(R_{m}, \sigma\right)}
\end{gathered}
$$

\section{EXPERIMENTS}

In our research, an $8 \times 8$ square meters indoor area as shown in Fig. 4 is used for the experiments. In order to test the superiority of IPS and fuzzy estimation method developed, the RSS signals for 288 points, 392 points, 440 points and 704 points by IPS system are collected. The sensing points of 288, 392, 440 and 704 are presented in Fig. 5(a) to Fig. 5(d), respectively.

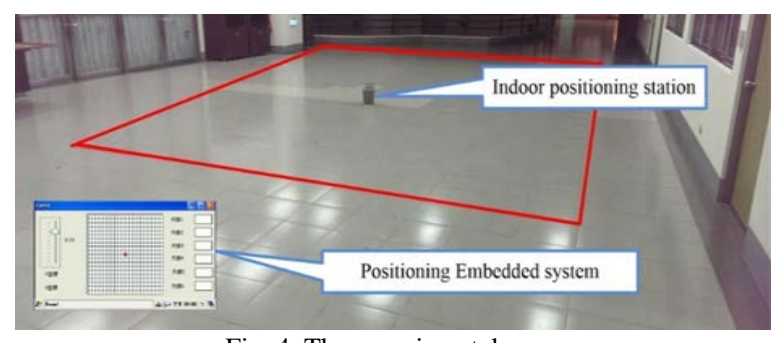

Fig. 4. The experimental area.

In order to decrease the noise disturbance caused by the unnecessary reference nodes, only ten reference nodes those have the closest RSS signals between reference node and 
blind node are selected for doing the positioning calculation. In other words, for each blind node, only 10 closest neighbor points are used in fuzzy positioning estimation.

In first experiment, 288 sensing points are used as the reference nodes of IPS, and then test the positioning accuracies of other three sets of blind nodes, i.e., 392 points, 440 points and 704 points. Table I lists the mean absolute errors of positioning estimations under the 288-based reference nodes.

TABLE I: THE Positioning ERRORS OF 392, 440 AND 704 PoINTS BASED ON 288 REFERENCE NODES

\begin{tabular}{|c|c|c|c|}
\hline \multirow{2}{*}{$\sigma$} & 392 points & 440 points & 704 points \\
\cline { 2 - 4 } & MAE $(\mathrm{cm})$ & MAE $(\mathrm{cm})$ & MAE $(\mathrm{cm})$ \\
\hline$\sigma=0.01$ & 49.6483 & 80.7379 & 50.1186 \\
\hline$\sigma=0.02$ & 45.9866 & 72.6805 & 46.8152 \\
\hline$\sigma=0.03$ & 44.1506 & 64.6079 & 45.7756 \\
\hline$\sigma=0.04$ & 45.2568 & 61.0226 & 48.2526 \\
\hline$\sigma=0.05$ & 47.7577 & 61.4149 & 51.9893 \\
\hline$\sigma=0.06$ & 50.7182 & 63.2862 & 55.5901 \\
\hline$\sigma=0.07$ & 53.4909 & 65.0631 & 58.5567 \\
\hline$\sigma=0.08$ & 55.6987 & 66.5014 & 60.7889 \\
\hline$\sigma=0.09$ & 57.3451 & 67.6033 & 62.4138 \\
\hline$\sigma=0.1$ & 58.5591 & 68.4348 & 63.5979 \\
\hline
\end{tabular}

\begin{tabular}{|c|c|c|c|c|c|c|c|c|c|c|c|c|c|c|c|c|}
\hline$\circ$ & & & & & & & $\bullet$ & $\bullet$ & $\bullet$ & & • & $\cdot$ & $\bullet$ & & & \\
\hline & $\bullet$ & • & • & . & • & • & $\bullet$ & • & • & - & • & - & • & & & - \\
\hline & • & • & • & 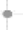 & • & • & • & • & • & • & $\bullet$ & • & • & $\bullet$ & & - \\
\hline & • & . & • & - & • & - & $\bullet$ & • & • & - & • & - & - & - & & \\
\hline & • & $\bullet$ & $\bullet$ & $\bullet$ & • & $\bullet$ & - & $\bullet$ & $\bullet$ & $\bullet$ & $\bullet$ & $\bullet$ & $\bullet$ & & & \\
\hline & $\bullet$ & : & $\bullet$ & $\bullet$ & • & $\bullet$ & - & : & $\bullet$ & • & $\dot{0}$ & : & • & • & & \\
\hline & . & $\bullet$ & . & $\bullet$ & $\bullet$ & $\bullet$ & . & . & $\bullet$ & . & . & . & & $\bullet$ & & \\
\hline & $\bullet$ & • & $\bullet$ & . & • & $\bullet$ & $\bullet$ & & $\bullet$ & • & • & $\bullet$ & • & $\bullet$ & • & $\bullet$ \\
\hline & . & • & - & • & • & • & $\bullet$ & - & • & $\bullet$ & • & • & $\bullet$ & $\bullet$ & & - \\
\hline & • & $\cdot$ & $\bullet$ & • & $\bullet$ & • & $\bullet$ & $\cdot$ & • & • & • & • & - & 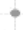 & & • \\
\hline & 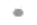 & • & $\bullet$ & • & $\bullet$ & • & • & $\bullet$ & $\bullet$ & - & • & • & $\bullet$ & $\bullet$ & & • \\
\hline$\bullet$ & • & • & $\bullet$ & • & - & 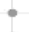 & - & $\bullet$ & $\bullet$ & • & $\bullet$ & • & • & & & • \\
\hline & : & $\bullet$ & $\bullet$ & 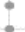 & $\bullet$ & $\bullet$ & 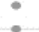 & $\bullet$ & $\bullet$ & $:$ & 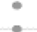 & $:$ & • & 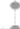 & & 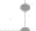 \\
\hline & & . & • & & $\bullet$ & $\bullet$ & • & $\bullet$ & $\bullet$ & $\bullet$ & $\bullet$ & . & & & & \\
\hline 0 & & 1 & & 2 & & & & & & 5 & & & & & & \\
\hline
\end{tabular}

(a)

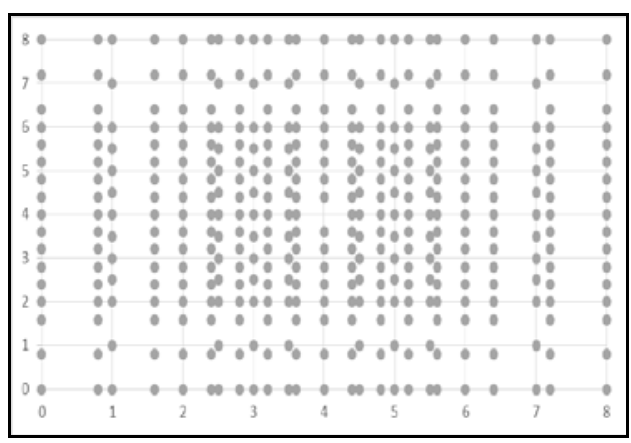

(b)

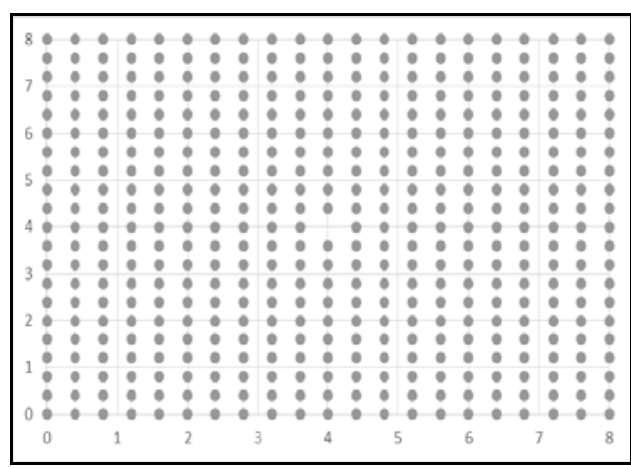

(c)

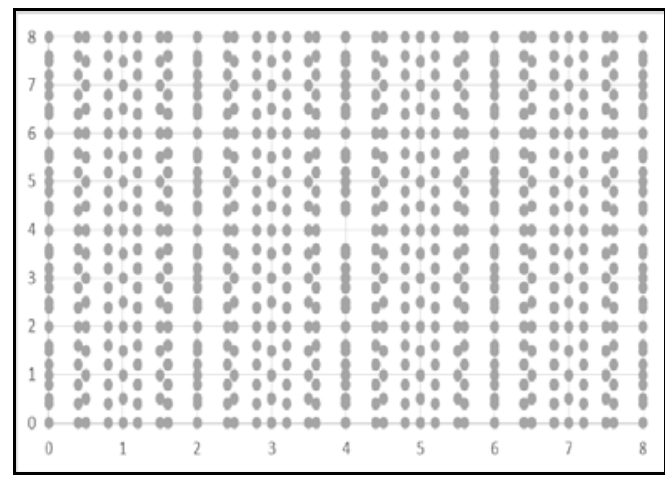

(d)

Fig. 5. The diagrams of sensing points.

In second experiment, 392 sensing points are used as the reference nodes, the other three sets of blind nodes, i.e., 288 points, 440 points and 704 points are tested. Table II lists the mean absolute errors of positioning estimations under the 392-based reference nodes.

TABLE II: The Positioning ERRoRs OF 288, 440 AND 704 POINTS BASED ON 392 REFERENCE NODES

\begin{tabular}{|c|c|c|c|}
\hline \multirow{2}{*}{$\sigma$} & 288 points & 440 points & 704 points \\
\cline { 2 - 4 } & MAE $(\mathrm{cm})$ & MAE $(\mathrm{cm})$ & MAE $(\mathrm{cm})$ \\
\hline$\sigma=0.01$ & 53.2433 & 38.3618 & 43.5007 \\
\hline$\sigma=0.02$ & 51.6871 & 37.5093 & 41.4810 \\
\hline$\sigma=0.03$ & 50.7746 & 39.4499 & 41.3907 \\
\hline$\sigma=0.04$ & 52.7392 & 45.2504 & 46.0379 \\
\hline$\sigma=0.05$ & 56.6001 & 52.0326 & 51.8982 \\
\hline$\sigma=0.06$ & 60.2394 & 57.7474 & 56.9473 \\
\hline$\sigma=0.07$ & 63.1585 & 61.8788 & 60.7150 \\
\hline$\sigma=0.08$ & 65.3008 & 64.7063 & 63.3549 \\
\hline$\sigma=0.09$ & 66.8318 & 66.6512 & 65.1977 \\
\hline$\sigma=0.1$ & 67.9398 & 68.0207 & 66.5085 \\
\hline
\end{tabular}

In third experiment, 440 sensing points are changed to be the reference nodes, the other three sets of blind nodes, i.e., 288 points, 392 points and 704 points are tested. Table III lists the mean absolute errors of positioning estimations under the 440-based reference nodes.

TABLE III: THE POSITIONING ERRORS OF 288, 392 AND 704 POINTS BASED ON 440 REFERENCE NODES

\begin{tabular}{|c|c|c|c|}
\hline \multirow{2}{*}{$\sigma$} & 288 points & 392 points & 704 points \\
\cline { 2 - 4 } & MAE $(\mathrm{cm})$ & MAE $(\mathrm{cm})$ & MAE $(\mathrm{cm})$ \\
\hline$\sigma=0.01$ & 71.0395 & 17.8032 & 30.4742 \\
\hline$\sigma=0.02$ & 65.0459 & 18.1327 & 29.6772 \\
\hline$\sigma=0.03$ & 59.1310 & 22.7253 & 32.3687 \\
\hline$\sigma=0.04$ & 58.1032 & 31.1503 & 39.1646 \\
\hline$\sigma=0.05$ & 59.2758 & 39.1492 & 46.2156 \\
\hline$\sigma=0.06$ & 60.8408 & 44.8615 & 51.4007 \\
\hline$\sigma=0.07$ & 62.3473 & 48.6798 & 54.9012 \\
\hline$\sigma=0.08$ & 63.5094 & 51.2720 & 57.2675 \\
\hline$\sigma=0.09$ & 64.3671 & 53.0657 & 58.8952 \\
\hline$\sigma=0.1$ & 65.0014 & 54.3311 & 60.0415 \\
\hline
\end{tabular}

In last experiment, 704 sensing points are the reference nodes, and the other three sets of blind nodes, i.e., 288 points, 392 points and 440 points are tested. Table IV lists the mean 
absolute errors of positioning estimations under the 704-based reference nodes.

From the experimental results shown, it is clearly found that the positioning errors become more and more accurate if more reference nodes are obtained. The best positioning MAE could reach to $0.0835 \mathrm{~cm}$ under 704-based reference nodes.

TABLE IV: THE POSITIONING ERRORS OF 288, 392 AND 440 POINTS BASED ON 704 REFERENCE NODES

\begin{tabular}{|c|c|c|c|}
\hline \multirow{2}{*}{$\sigma$} & 288 points & 392 points & 440 points \\
\cline { 2 - 4 } & MAE $(\mathrm{cm})$ & MAE $(\mathrm{cm})$ & MAE $(\mathrm{cm})$ \\
\hline$\sigma=0.01$ & 4.1529 & 0.0835 & 3.1418 \\
\hline$\sigma=0.02$ & 8.3464 & 2.4429 & 7.3441 \\
\hline$\sigma=0.03$ & 21.1941 & 12.2807 & 19.1347 \\
\hline$\sigma=0.04$ & 34.0465 & 24.3425 & 32.0262 \\
\hline$\sigma=0.05$ & 42.3385 & 33.4878 & 41.3185 \\
\hline$\sigma=0.06$ & 47.3073 & 39.5509 & 47.2433 \\
\hline$\sigma=0.07$ & 50.4972 & 43.4366 & 50.9298 \\
\hline$\sigma=0.08$ & 52.6107 & 45.9678 & 53.2863 \\
\hline$\sigma=0.09$ & 54.0444 & 47.6720 & 54.8571 \\
\hline$\sigma=0.1$ & 55.0490 & 48.8594 & 55.9480 \\
\hline
\end{tabular}

\section{CONCLUSION}

This research presents a sectored antenna array indoor positioning system by using fuzzy estimation algorithm. Four experiments were executed based on different reference database. From the experimental results shown, we found that the positioning errors performed by fuzzy estimator become more accurate under the case of more reference nodes are obtained. Besides, in each experiment, the positioning accuracy is highly related to the $\sigma$ vale. In fact, such a situation is foreseeable. It means the closer neighbor point should have larger weight and play more important role in the positioning performance. We believe that the positioning accuracy could be greatly improved if there are more reference nodes could be obtained. It also means that the proposed IPS system developed do have more potential in its real application.

\section{REFERENCES}

[1] Y. Y. Gu, A. Lo, and I. Niemegeers, "A survey of indoor positioning systems for wireless personal networks," IEEE Communications Surveys \& Tutorials, vol. 11, no. 1, pp. 13-32, 2009.

[2] H. Liu, H. Darabi, P. Banerjee, and J. Liu, "Survey of wireless indoor positioning techniques and systems," IEEE Trans. on Systems, Man, and Cybernetics, vol. 37, no. 6, pp. 1067-1077, 2007.

[3] G. W. Shi and Y. Ming, "Survey of indoor positioning systems based on ultra-wideband (UWB) technology," Lecture Notes in Electrical Engineering, Wireless Communications, Networking and Applications, pp. 1269-1278, vol. 348, 2016.

[4] B. Kim, W. Bong, and Y. C. Kim, "Indoor localization for Wi-Fi devices by cross-monitoring AP and weighted triangulation,” in Proc. IEEE Consumer Communications and Networking Conference, 2011, pp. 933-936.

[5] M. Vossiek, L. Wiebking, P. Gulden, J. Wiehardt, C. Hoffmann, and P. Heide, "Wireless local positioning," IEEE Microwave Mag., vol. 4, no. 4, pp. 77-86, 2003.

[6] G. Nuno-Barrau and J. M. Paez-Borrallo, "A new location estimation system for wireless networks based on linear discriminant functions and hidden Markov models," EURASIP J. Applied Signal Processing. vol. 1, no. 1, pp. 1-17, 2006.

[7] M. Brunato and K. K. Csaba, "Transparent location fingerprinting for wireless services," in Proc. Med-Hoc-Net, 2002.

[8] J. Krumm, S. B. Harris, B. Meyers, B. Brumitt, M. Hale, and S. Shafer, "Multi-camera multi-Person tracking for easy living," in Proc. 3rd
IEEE Int'l Workshop Visual Surveillance, IEEE Press, Piscataway, 2000.

[9] D. Focken and R. Stiefelhagen, "Towards vision-based 3-D people tracking in a smart room," in Proc. 4th IEEE Intl Conference on Multimodal Interfaces, 2002, pp. 400-405.

[10] T. Roos, P. Myllymaki, H. Tirri, P. Misikangas, and J. Sievanan, “A probabilistic approach to WLAN user location estimation," Int. J. Wireless Inf. Netw., vol. 9, no. 3, pp. 155-164, 2002.

[11] V. Honkavirta, T. Perala, S. Ali-Loytty, and R. Piche, R. "A comparative survey of WLAN location fingerprinting methods," in Proc. 6th Workshop on Positioning, Navigation and Communication, 2009, pp. 243-251.

[12] M. Y. Umair, K. V. Ramana, D. K. Yang, "An enhanced K-Nearest Neighbor algorithm for indoor positioning systems in a WLAN," in Proc. 2014 IEEE Conference on Computers, Communications and Its Applications, 2014, pp. 19-23.

[13] K. F. S. Wong, I. W. Tsang, V. Cheung, S. H. G. Chan, and J. T. Kwok, "Position estimation for wireless sensor networks," in Proc. IEEE Global Telecommunications Conference, 2005, pp. 2772-2776.

[14] C. Savarese, J. M. Rabaey, and J. Beutel, "Locationing in distributed ad-hoc wireless sensor networks," in Proc. IEEE ICASSP, 2001, pp. 2037-2040.

[15] H. D. Chon, S. Jun, H. Jung, and S. W. An, "Using RFID for accurate positioning,” Journal of Global Positioning Systems, vol. 3, pp. 32-39, 2004.

[16] H. L. Ding, W. W. Y. Ng, P. P. K. Chan, D. L. Wu, X. L. Chen, and D. S. Yeung, "RFID indoor positioning using RBFNN with L-GEM," in Proc. IEEE 2010 International Conference on Machine Learning and Cybernetics, 2010, pp. 1147-1152.

[17] A. K. M. M. Hossain, W. S. Soh, "A comprehensive study of Bluetooth signal parameters for localization," in Proc. 18th Annual IEEE International Symposium on Personal, Indoor and Mobile Radio Communications, 2007, pp. 1-5.

[18] F. Subhan, H. Hasbullah, A. Rozyyev, and S. T. Bakhsh, "Indoor positioning in Bluetooth networks using fingerprinting and lateration approach," in Proc. 2011 International Conference on Information Science and Applications, 2001, pp. 1-9.

[19] W. P. Chen and X. F. Meng, "A cooperative localization scheme for Zigbee-based wireless sensor networks," in Proc. 14th IEEE International Conference on Networks, pp. 1-5, 2006.

[20] G. Goncalo and S. Helena, "Indoor location system using ZigBee technology," in Proc. Third International Conference on Sensor Technologies and Applications, 2009, pp. 152-157.

[21] C. Y. Chen, Y. U. Chen, S. W. Chen, C. Y. Shen, and R. C. Hwang, "A fuzzy indoor positioning system with ZigBee wireless sensors," Journal of Electrical and Electronic Engineering, vol. 4, no. 5, pp. 97-102, 2016

[22] A. Rozyyev, H. Hasbullah, and F. Subhan, "Combined k-nearest neighbors and fuzzy logic indoor localization technique for wireless sensor network,” J. Inf. Technol. Res., vol. 4, pp. 155-165, 2012.

[23] A. K. Singh, N. Purohit, and S. Varma, "Fuzzy logic based clustering in wireless sensor networks: a survey, "Int. J. Electron. vol. 100, pp. 121-141, 2012.

[24] S. Onofre, B. Caseiro, J. P. Pimentão, and P. Sousa, "Using fuzzy logic to improve BLE indoor positioning system, Technological Innovation for Cyber-Physical Systems," in Proc. 7th IFIP WG 5.5/SOCOLNET Advanced Doctoral Conference on Computing, Electrical and Industrial Systems, pp 169-177, 2016.

[25] O. Akeem, O. M. T. Abu, and M. Teddy, "Fuzzy logic based compensated Wi-Fi signal strength for indoor positioning," in Proc. the 2013 International Conference on Advanced Computer Science Applications and Technologies, 2013, pp. 444-449.

[26] M. Alakhras, M. Oussalah, and M. I. Hussein, "Fuzzy inference with parameter identification for Indoor WLAN positioning," in Proc. the IAENG World Congress on Engineering, 2015, vol. 1, pp. 641-648.

[27] A. Teuber and B. Eissfeller, "A two-stage fuzzy logic approach for wireless LAN indoor positioning," in Proc. IEEE/ION Position, Location and Navigation Symposium, pp. 730-738, 2006.

[28] C. Y. Chen, Y. J. Chen, Y. C. Weng, and R. C. Hwang, "A neural-network-based indoor positioning system using sectored antenna array, "International Journal of New Technology and Research (IJNTR). vol. 2, no. 3, pp. 26-29, 2016.

[29] Y. C. Weng, "Design of sector array antenna for indoor positioning with modified probabilistic neural network," Master Thesis, Department of Computer and Communication, Shu-Te University, Kaohsiung, Taiwan, 2014.

[30] L. X. Wang et al., A Course in Fuzzy Systems and Control, Prentice Hall Inc., 1997. 


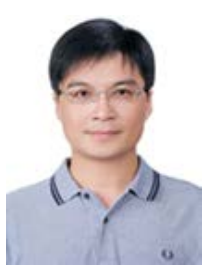

Chih-Yung Chen is an assistant professor of Department of Computer and Communication at Shu-Te University, Taiwan. He received his B.S and M.S. degrees in computer science and information engineering from Tamkang University in 1998 and 2000 respectively, and the Ph.D. degree of electrical engineering from I-Shou University in 2008. His current research interests are artificial intelligence, autonomous robot control, database system and indoor positioning technologies.

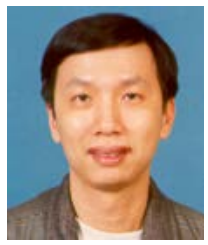

Shen-Whan Chen received his B.S. degree in electrical engineering from Tsing-Hwa University, Taiwan in 1980, M.S. degrees in electrical engineering from Marquette University, USA in 1987, and Ph.D. degrees in electrical engineering from university of Illinois at Chicago, USA in 1994. He has worked for Delco, MA/COM, Hughes Space and Comm., etc. He is currently with the Communication Engineering Department, I-Shou University at Kao-Shung, Taiwan as an associate professor. His main research interests are in the RF circuit design for wireless communication and AI signal processing.

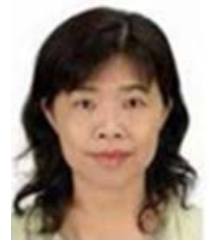

Yu-Ju Chen currently is an assistant professor of Information Management Department at Cheng Shiu University. Her research interests include artificial intelligence, fuzzy theory and information management.

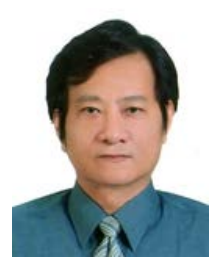

Rey-Chue Hwang received his Ph.D. degree in electrical engineering from Southern Methodist University, Dallas, TX, USA in 1993. Currently, he is a full professor of Electrical Engineering Department, I-Shou University, Taiwan, R.O.C. Dr. Hwang is a fellow of IET and a senior member of IEEE. He chartered IEEE CIS Chapter, Tainan Section and served as the co-chair and chair from year 2004 to year 2009 His research interests include the areas of artificial intelligent system, signal processing and fuzzy control. 